\section{Enhanced Machine Controller Architecture Overview}

Frederick M. Proctor

Systems Integration Group

John Michaloski

Intelligent Controls Group

U.S. DEPARTMENT OF COMMERCE Technology Administration

National Institute of Standards and Technology

Robot Systems Division

Bldg. 220 Rm. B124

Gaithersburg, MD 20899 



\section{Enhanced Machine} Controller Architecture

\section{Overview}

Frederick M. Proctor

Systems Integration Group

John Michaloski

Intelligent Controls Group

U.S. DEPARTMENT OF COMMERCE

Technology Administration

National Institute of Standards

and Technology

Robot Systems Division

Bidg. 220 Rm. B124

Gaithersburg, MD 20899

December 1993

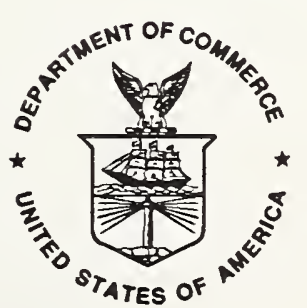

U.S. DEPARTMENT OF COMMERCE Ronald H. Brown, Secretary

TECHNOLOGY ADMINISTRATION

Mary L Good, Under Secretary for Technology

NATIONAL INSTITUTE OF STANDARDS

AND TECHNOLOGY

Arati Prabhakar, Director 



\section{ENHANCED MACHINE CONTROLLER}

\section{ARCHITECTURE OVERVIEW}

Fred Proctor

John Michaloski

Manufacturing Engineering Laboratory

National Institute of Standards and Technology 
This work was performed by U.S. Government employees as part of their official duties, and is not subject to copyright. 


\section{Introduction}

This overview provides a brief summary of NIST's efforts to define an Enhanced Machine Controller (EMC) architecture, with emphasis on the requirements of machine tools. The primary concern is to implement an open architecture conforming to the Next Generation Controller Specification for an Open System Architecture Standard (NGC SOSAS) [1] and to the NIST Realtime Control System reference model architecture (RCS) $[2,3]$ by defining a set of modules, their interfaces, and required basic functionality. The importance of multi-level feedback, "art-to-part" requirements, and sensor-based enhancements will be directly addressed during the development of the architecture.

Section 2 describes the machine tool hardware and software systems that are being implemented at NIST for the EMC project. This section was included at the beginning of the document so that the subsequent discussions could be rooted in real machine control scenarios. Section 3 details the goals of the EMC project and how the systems being implemented will help meet those goals. Section 4 lists the requirements of users and systems integrators which drive the development of the architecture. Section 5 outlines the EMC architecture and its relationships to the existing NIST hierarchical control architecture and current industry standardization efforts. Sections 6 through 8 detail the modules which make up the controller. Section 9 details the controller infrastructure (the "Virtual Machine"), Section 10 outlines the safety issue, and Section 11 provides a road map for the subsequent documents that will provide a more complete and detailed description of the EMC architecture.

Disclaimer: No approval or endorsement of any commercial product by the National Institute of Standards and Technology is intended or implied. Certain commercial equipment, instruments, or materials are identified in this report in order to facilitate understanding. Such identification does not imply recommendation or endorsement by the National Institute of Standards and Technology, nor does it imply that the materials or equipment identified are necessarily the best available for the purpose.

\section{System Description}

The EMC controller implementation efforts at NIST are divided between two platforms, the Shop Floor Controller and the Laboratory Development Controller. The Shop Floor Controller is based on the PMAC motion controller from Delta Tau Data Systems, in an environmentally-enclosed 486 IBM-PC. The 486 is running the Microsoft MS-DOS operating system. This controller is connected to a Monarch VMC-75 3-1/2 axis vertical machining center, which has a positioning (W) axis and a tool changer. A VGA monitor, keyboard, and trackball were mounted into an enclosure for the operator console, replacing the original General Electric Mark Century 2000 controller console. In addition, the control console includes jog buttons, a handwheel, and mode select switches. All the Monarch control input and output lines run into a terminal block inside a cabinet at the back of the machine, and are interfaced to the PMAC motion controller using a variety of interconnect modules provided by Delta Tau.

In the Shop Floor Controller, the host computer is running Delta Tau's PMAC-NC program. This program provides operator interface functions such as graphics display and control panel input, manages data such as tool offsets and parameter tables which affect axis motion, and manages the rotary buffer used to download part programs to the PMAC board. NIST developed graphics "CNC front ends" for this controller which can emulate either the Fanuc 10, GE 2000, or Allen-Bradley 8200 commercial controller interfaces. EIA-274-D part programs are first parsed by the PMACNC program, and translated into the PMAC native language. The PMAC board communicates to the host PC using a dual-ported RAM mechanism. The PMAC board performs the coordinate 
system trajectory planning, the axis trajectory interpolation, the servo computations, all of the PLC tasks (selection between $\mathrm{Z}$ or W axes, control panel I/O, and tool changer), and axis limit checking. The Laboratory Development Controller is also hosted by a 486 PC, and uses the PMAC motion controller as well. However, this system differs in several ways. Most importantly, it is not connected to a production machine tool, but to a hardware simulator which contains DC servomotors and encoders. This allows development work to proceed without anxiety about injury or damaging expensive production machinery. The Laboratory Development Controller is running a real-time Unix operating system which is POSIX [4] compliant, and is controlled by software from Cimetrix, Inc. Most of the functions performed by the PMAC board in the Shop Floor Controller are performed in software on the PC host, namely PLC programs, part program interpretation, and trajectory generation. Both systems are capable of handling EIA-274-D programs, which can be generated off-line using a CAD/CAM system. A recent benchmark demonstrated both controllers running the NAS-979 "circle diamond square" test part program.

\section{Implementation Plan}

This document serves as an initial reference for the architecture, module definitions, and interfaces which are being prototyped and implemented on both the Shop Floor Controller and Laboratory Development Controller. Following this document will be the detailed specifications of each of the modules laid out here, whose exact contents will be driven by the lessons learned during the prototyping and implementation phases with both controllers. Implementation and prototyping occur according to the following plan. Integration of existing enhancements, such as thermal compensation and part probing, are performed on the Shop Floor Controller, because these enhancements require actual analysis on the machine tool. Once these enhancements are demonstrated and hardened, they are ported to the Laboratory Development Controller for "wrapping" as modules which conform to the SOSAS architecture. These wrapped enhancements are then be demonstrated again on the Monarch for a demonstration of a fully SOSAS-conformant controller. Additional investigations which do not require machine tool time are performed on the Laboratory Development Controller from the beginning. Such investigations include the testing of new operating systems, CAD/CAM interfaces, and interfaces to the factory.

Throughout this document, it should be understood that NIST's role in the EMC is not to act as a developer of third-party enhancements, but as a system integrator whose intent is to take enhancements already developed by third parties, port them to the controller implementations, and document the effort required to do this. The result will be a set of interfaces required to make this task easier on systems integrators.

\section{EMC Requirements}

The following sections outline the development of the EMC architecture, using an analysis of the requirements placed on the architecture from several areas.

\subsection{Legacy Requirements}

The EMC must provide at least the capabilities found in the legacy systems familiar to machinists today. These legacy capabilities, as applicable to the NIST testbed, are:

- three-axis contour milling;

- automatic tool changing;

- EIA-274-D part program interpretation;

- presentation of a graphic display typified by that of current controller technology; 
- "hands on" access by the machinist to physical jog buttons, wheels, and mode select switches.

\section{2 "Art-to-Part" Requirements}

Automatically generating a process plan based on CAD part descriptions obviously requires great system sophistication. For each step in a process plan, tool path planning is responsible for directing the machine tool cutter to remove designated volumes of material. The path planning generates motion primitives based on these volumes. It must account for proper machine tool setup and fixturing, and account for any fixturing interference. Each motion primitive must be transformed into motion segment trajectories. These segments are then transformed into a series of set points for the individual axes. At a coarse level of description, the system functionality can be loosely codified by the following breakdown:

- general purpose, such as computer-aided design or user interfacing (e.g., pendants, touch screens, or display graphics);

- part design, such as geometric calculation, STEP data handling, or data management;

- process planning and scheduling;

- elementary machining operations, such as Material Removal Surface Element Volume (MRSEV) or Boundary to B-Rep Conversion functions;

- tool path generation, such as NC programming interfaces, NC-code generation, and machining simulation.

\subsection{Feedback Requirements for Adaptive Control and Error Compensation}

While the machine tool's axis positions are closed with servo loops, typical NC programs are interpreted "open loop" with no process feedback for monitoring machining. Error compensation, if done at all, is done statically, by preprogramming instances where one would expect errors and then compensating for the errors. This is common in today's controllers which use lead screw compensation tables. Dimensional accuracy and part finish can be improved by incorporating dynamic closed-loop error compensation techniques, which resolves errors through environment sensing.One can attach a sensor to the problem area, read real-time sensor feedback, evaluate this reading against the expected model and then modify the course of action based on any measured error. Among the types of feedback and error compensation the EMC project will be exploring include:

- thermal error compensation

- geometric error compensation

- chatter control

- control algorithm error

One of the main criticisms of the SOSAS is that it did not grow out of a comprehensive prototyping effort. The EMC philosophy is to attempt to install the above enhancements to our shop floor and laboratory development controllers, and to supplement the SOSAS in areas where it says nothing about how this is to be done.

\subsection{Modularity, Portability, Extensibility, and Scalability Requirements}

Additional requirements are placed on the controller by users or system integrators who want to piece together their own systems component by component, modify the way their controller does certain things, apply their modifications to another controller, or start small and upgrade as they 
grow. These requirements mean that the controller architecture must be modular, extensible, portable, and scalable. Each of these is described in more detail in the following sections.

\subsubsection{Modularity}

Modularity refers to the ability of controls users and system integrators to purchase and replace components of the controller without unduly affecting the rest of the controller. For the EMC, this means the ability to replace hardware boards and peripheral devices such as disks or RAM with equivalent products from another source. The key to doing this is the definition of the modules which make up a controller, their functionality, their interfaces, and the basic controller infrastructure (bus, microprocessor, operating system, etc.) which can support the modules. The first three are addressed by the SOSAS but need to be fleshed out and validated; the infrastructure definition is necessarily a choice made by the system integrators, in this case NIST.

\subsubsection{Extensibility}

Extensibility refers to the ability of knowledgable users and third parties to incrementally add functionality to a module without replacing it completely. For example, an extensible plan interpreter would allow users to add statements to the planning language (such as a new G code). An extensible trajectory generator would allow this $\mathrm{G}$ code to be executed, while an extensible discrete input/output module would allow the easy addition of a new actuator or sensor. Extensibility is required by NIST for the incorporation of enhancements, but developing modules which contain the "hooks" for extensibility remains the responsibility of module vendors.

\subsubsection{Portability}

Portability refers to the ease with which a module can be made to run in a controller based on another platform. Portability normally refers to software source code, which should be compilable for a variety of CPUs and operating systems. Standards such as ANSI C and POSIX serve as a reference to which programmers adhere. For the EMC, portability is mostly an issue left to vendors with whom NIST is working, since little if any porting effort in the form of source code modification should be required of machine tool systems integrators.

\subsubsection{Scalability}

Scalability, like portability, refers to the ease with which a module can be made to run in a controller based on another platform, but unlike portability, scalability is an attribute of the controller architecture, not the implementation of the module. Scalability means that a controller based on the architecture may be implemented equally easily by systems integrators entirely in software on a fast processor, as a distributed multi-processor bus system, or in a standalone PC. Scalability of the architecture gives system integrators the ability to build controllers around their customer's needs and budget, and gives customers the freedom to "scale up" to a higherperformance implementation when their needs and budgets increase. Scalability is provided by the SOSAS, and will be validated in the EMC testbed.

\section{EMC Architecture}

This section provides a summary of the architecture, at a block-diagram level. The evolution of the architecture from its basis on the NIST RCS is discussed in the first section. The question of how the EMC architecture is related to the SOSAS is answered in the second section. The subsequent sections overview the modules which make up the EMC and the nature of the messages that are understood by each module. 


\subsection{EMC and the NIST Real-time Control System}

The EMC architecture is derived from the NIST Real-time Control System reference model architecture (RCS) [2] and NASREM, the NASA/NBS reference model architecture [3]. RCS builds upon experience acquired in the Automated Manufacturing Research Facility [5] during the 1980's for constructing hierarchies of controllers for machining workcells which include robot, machine tool, and coordinate measuring machines as components. The NASREM reference model places heavy emphasis on teleoperation, and puts a burden on the architecture to support real-time feedback from a human operator throughout the control hierarchy.

The EMC architecture conforms to both the NIST RCS reference model and to the Next Generation Controller Specification for an Open System Architecture Standard (NGC SOSAS), which is the specification resulting from the program sponsored by the U.S. Air Force for standardizing machine controllers. The NIST and SOSAS models differ mainly in two ways. First, RCS/NASREM specifies control modules that are arranged in a hierarchy, each module having the capability for closed-loop control; the SOSAS specifies no such hierarchy for control, although it does indicate a hierarchical layering of interfaces to the physical hardware level. Second, RCS/NASREM specifies a higher resolution for the definition of the control modules than does the SOSAS.

Since RCS/NASREM is more strict in its definition than is the SOSAS, defining a controller architecture which satisfies both is straightforward: one focuses on the stricter model, deriving an architecture whose components are then grouped and labeled according the coarser model. In the discussions that follow, nomenclature from both RCS/NASREM and the SOSAS will be used, so that a mapping between them will emerge.

\subsection{EMC and the SOSAS}

Following the SOSAS, the EMC defines the following Standardized Applications (SAs): Workstation Planning Standardized Application (WPSA), Workstation Management Standardized Application (WMSA), and Controls Standardized Application (CSA). As prescribed by the SOSAS, the EMC Standardized Applications communicate by passing Neutral Manufacturing Language (NML) messages. Communication within a single SA is not governed by the SOSAS, and is not required to use NML. Furthermore, the SOSAS does not place the individual SAs in a hierarchy, nor does it specify the architecture within the SAs other than defining the NML messages a particular SA must respond to and what that response should be. However, the SOSAS does not preclude organizing the SAs hierarchically in a particular implementation, nor does it disallow further refinement within the SAs. In the EMC, this organization and refinement is done so that the benefits of the NIST Real-time Control System architecture can be applied to machine control within the broad constraints of the SOSAS. The EMC organization places the WPSA, WMSA, and CSA into a hierarchy, and further refines the CSA into four modules which form a branched hierarchy as specified in RCS/NASREM.

We allow for Domain-Independent Applications (DIAs), as prescribed by the SOSAS, which let third parties enhance a controller by providing a function not present in the off-the-shelf version. The desire to incorporate enhancements as DIAs is a direct driver for an open architecture. DIAs can be a simple as a data logger which accumulates the time each tool has been used, or as complex as model-based thermal compensation. Perhaps the most important DIA is the operator interface, which is not part of the controller per se, but acts as an independent application allowing the user to view the state of the workstation and at times giving one direct control as specified in NASREM.

The Virtual Machine comprises the software which interfaces directly to the underlying controller hardware. The rationale for the VM is that something is needed to insure portability of SAs and 
modules across different operating systems and hardware platforms. The bulk of the VM functions are provided by the underlying operating system (e.g., the file system, process tasking, interrupt servicing), but interfaces to these functions require standardization by the VM. The VM further standardizes the additional interfaces to the machine hardware, graphics displays, control panel interface, and other machine-specific functions.

\subsection{EMC Standardized Applications and Modules}

Each of the Standardized Applications, and the modules which make up the Controls Standardized Application, will be documented in the following sections so that these two questions can be answered: "What is it that the Standardized Application or module must do?", and "What does the Standardized Application or module have at its disposal in order to do it?"

The answer to the first question is determined by the messages that an SA or module accepts. These messages may come from another SA or module in the workstation, or from a DIA. A block diagram of the modules and their relationships is shown in Figure 1. The body of this diagram uses nomenclature from the SOSAS; in the left margin is the mapping to the NIST RCS architecture.

\subsection{EMC Communication and Messages}

The answer to the second question is determined by what messages the SA or module can issue. These messages are directed to another SA or module in the workstation, or to the Virtual Machine. A depiction of the message pathways and the types of messages they convey is shown in Figure 2 below. The messages which flow between SAs constitute NML, which is specified by the SOSAS. The messages which flow inside the CSA between modules conform to the RCS and NASREM reference models and will be fully defined as part of the EMC development.

\section{The Workstation Planning Standardized Application (WPSA)}

The WPSA is responsible for making a part design and generating the control plan which will transform an "as-is" workpiece, typically a blank, into a "to-be" part. The control plan handles part handling, set-up, and fixturing, and then identifies the sequence of operations for the workstation"s devices. A workstation may be a simple as a three-axis vertical machining center, or a complex station containing a machining center, part handling robot, and inspection system. In both cases, the WPSA allows the development of control plans for running the workstation. In a simple setup, planning may involve the generation of NC programs by a PC-based CAD/CAM system. In more complicated scenarios, the WPSA may rely on scheduling and process planning software, solid modelers, and expert systems to automatically produce a complex part requiring machining on several tools and robot part handling and assembly. In the RCS architecture, the WPSA corresponds to the Cell level.

WPSAs of today typically sit upstream of the controller, and allow CAD programmers to graphically generate IGES files for their parts. CAM systems and post processors let them take their IGES files and generate cutter location (CL) data and ultimately NC code for a particular machine. Usually, no information from downstream production is automatically fed back into the CAD/CAM system: planning runs "open loop." For example, if the programmer specifies a 2-inch end mill during the NC code generation phase, and only a $1 \frac{3}{4}$-inch end mill is available at run time, the part programmer is required to repeat that phase and insert more milling passes.

Giving the WPSA feedback from the controller allows for more intelligent machining. For instance, it is not difficult to imagine the tool mismatch reprogramming described above being automatic: at run time, when a tool mismatch is detected, a message is sent to the CAM portion of the WPSA to regenerate the NC code based on the existing tools. 


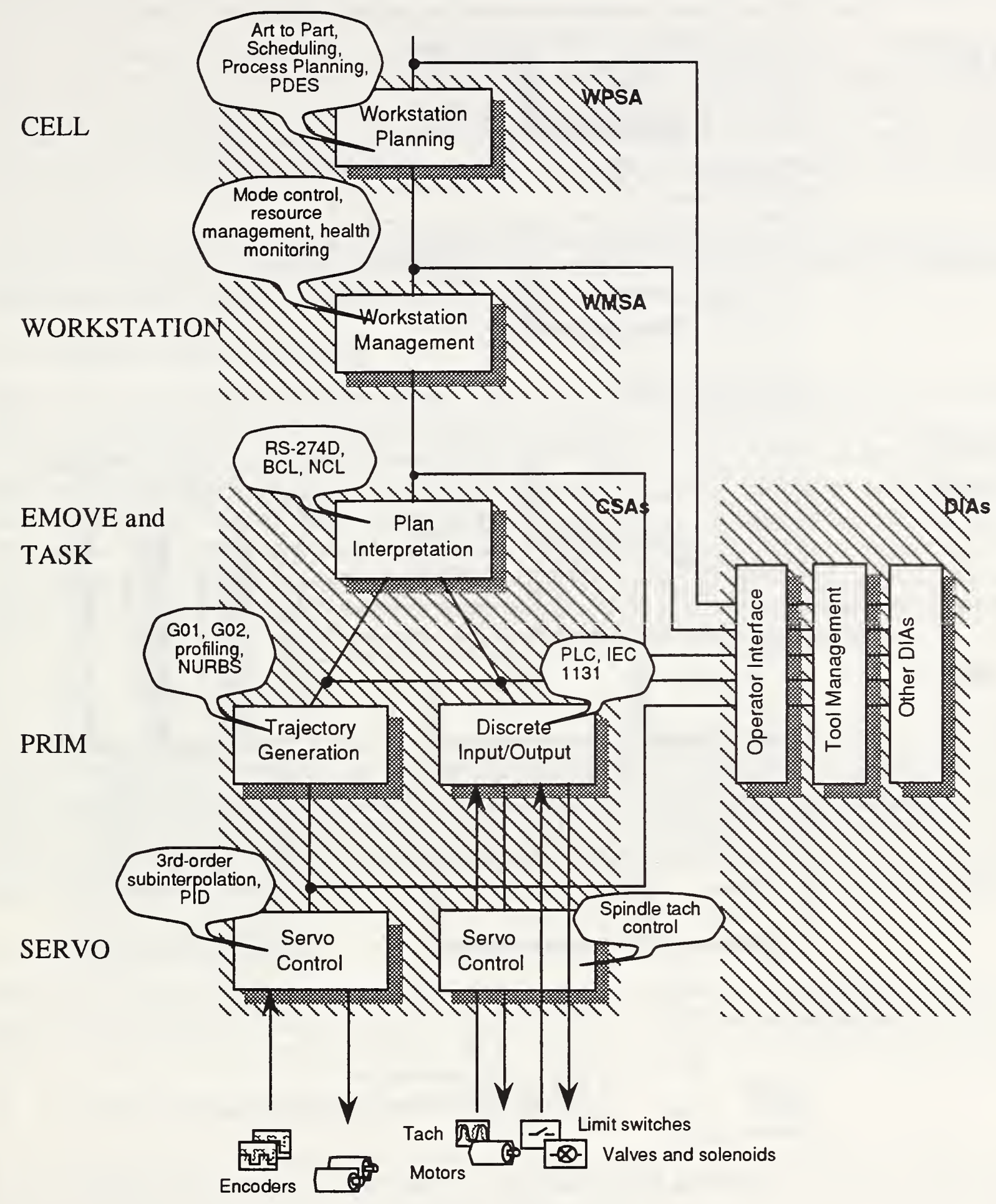

Figure 1. EMC Modules

The following sections outline the capabilities of the WPSA that will be implemented in the EMC under the current program, or have been implemented at NIST in the past on other RCS controllers and are candidates for porting to the EMC platforms in the future. 


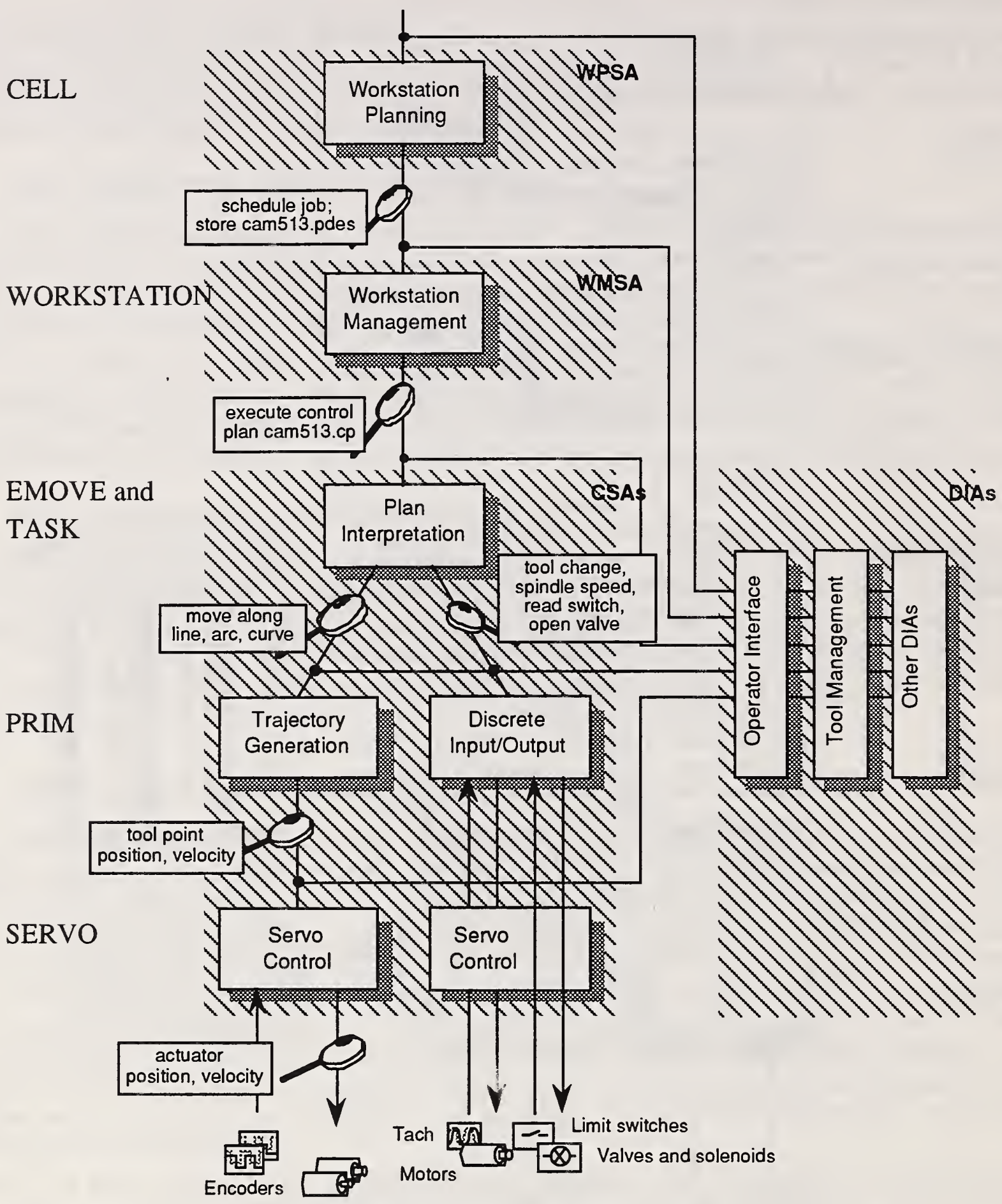

Figure 2. EMC Messages

\subsection{CAD/CAM Design and Planning}

The EMC must handle at least the functionality provided by "legacy systems." Such systems are the typical CNC machining centers which are programmed in some dialect of EIA-274-D, and exist in a shop where programmers seated at workstations running commercial CAD/CAM software can generate NC code using some collection of post processors tailored to each machine. 
The programs are generated off-line, and are punched onto tape or downloaded over a network for execution. Operators can single step the programs during machining, or edit them.

The NC code generated in such a scenario is analogous to a SOSAS Control Plan. The WPSA must either generate EIA-274-D, BCL, or NCL NC code. Current CAD/CAM systems can output EIA-274-D, and NIST is using such systems from a variety of vendors, including Alpha-1, CADKEY, MasterCAM, and AutoCAD. NIST has developed a translator which converts EIA-274-D into NCL (but not the reverse, as NCL contains instructions that are not contained in EIA-274-D).

NC verification tools are supported by the EMC WPSA. At the Shop Floor Controller console, the operator can bring up a standalone commercial product, N-See, and watch as the EIA-274-D code is interpreted and a solid model of the stock is machined graphically.

\subsection{Solid and Surface Modeling}

Some of the CAD/CAM systems listed above provide solid modeling capabilities. For example, NIST has purchased the solid modeling option to AutoCAD. The Alpha-1 CAD/CAM system uses a novel surface construction method for describing parts, lacing the surfaces together at edges which are represented as non-uniform rational B splines (NURBS). The addition of NURBS to the primitives used to describe edges means that complex shapes can now be described exactly, without resorting to approximation using small segments of straight lines or circular arcs. The result of this is a reduction in control plan size, a reduction in the time it takes to interpret a control plan, and an improved surface finish. For these benefits to be realized, however, modifications in both the Plan Interpretation and Trajectory Generation modules need to be made.

\subsection{Feature Recognition}

Previous work in the NIST Automated Manufacturing Research Facility resulted in an NC code generator which took a feature-based description of the part to be machined and output EIA-274-D code in the dialect used on the Monarch VMC-75's previous controller, a General Electric Mark Century 2000 [6]. Since both the Shop Floor Controller and Laboratory Development Controller platforms accept EIA-274-D, this package will port to the new systems with the minimum effort required to change the output format from the GE-2000 dialect to standard EIA-274-D.

\subsection{PDES Data Interfacing}

Future work includes augmenting the WPSA so that it handles geometry and process planning data represented in PDES [7]. This will involve cooperation with vendors of CAD/CAM and process planning software. This cooperation is currently taking place as part of the NIST PDES testbed.

\subsection{Coordinated Planning Between Multiple Devices}

The WPSA will handle the generation of control plans for multiple devices (e.g., multiple CSAs) in a single workstation, such as a workstation in which a robot loads a machine tool. No scheduling or process planning software is currently used in the EMC, since only one CSA is used to control the machining center. However, with the incorporation of a standard interface to the factory (cell), this could be done, particularly since both the Shop Floor Controller and Laboratory Development Controller are connected to local area networks.

Note that current planning languages, such as EIA-274-D, do not have language support for multiple devices directly. Synchronization between multiple devices, e.g., a robot and machine tool, is typically done using handshaking via interlocks. These handshaking statements are programmed into both the robot and machine tool programs explicitly. It is the job of the WPSA 
to generate such interlocking in plans developed for multiple devices controlled by separate CSAs. If tight synchronization is required, those devices must be controlled in a single CSA, as described in a later section.

\section{The Workstation Management Standardized Application (WMSA)}

The WPSA communicates through message passing to the WMSA by passing NML messages. The WMSA is responsible for managing the state of the workstation, and performs the functions of mode control, resource management, health monitoring, and other administrative tasks. In simple workstations, the WMSA may simply control the interaction of a machinist and the part program, disabling certain modes at times for safety or part integrity. In complex workstations, the WMSA may perform startup, shutdown, and safety operations, monitor levels of consumables, lock out resources shared by robots and machine tools, and maintain a database of machine operations and resource usages. In the RCS architecture, the WMSA corresponds to the Workstation level.

The WMSA can be regarded as the main "event loop" of the workstation, processing events from the operator, factory cell interface, or sensors, switching between modes and states, running control plans, logging data, switching control to the machinist, and performing many other functions. It is the WMSA which coordinates the actions of the other physical machines, such as machine tools and robots, which cooperate in a machining cell.

The SOSAS identifies 17 behaviors of the WMSA:

1. handle NML messages

2. perform workstation startup sequence

3. perform external communication

4. control workstation execution

5. handle exceptions

6. perform shutdown sequence

7. configure workstation

8. control mode/state

9. monitor safety

10. manage health

11. control diagnostics

12. schedule tasks

13. determine resource availability

14. handle resource request

15. manage application configuration

16. provide data logging

17. generate OBIOS calls

The following sections group these 17 behaviors into areas that we will address in the EMC.

\subsection{WMSA Mode and State Control}

The WMSA is required to maintain and switch between the operating modes of the workstation. The SOSAS identifies five modes:
1. startup
2. normal production
3. failure recovery 
4. maintenance

5. shutdown

Within each mode there are states that require WMSA maintenance. For example, in each of the five modes there must be some provision for operator override, implying that there are an automatic and a manual state. In normal production mode, there may be automatic, manual, or manual data input states in which the operator has varying degrees of control during machining.

\subsubsection{Execution States}

States of operation maintained by the WMSA include manual and automatic. The manual state of normal production mode allows the machinist to control the operation of the machine using the operator interface. This includes such actions as homing and jogging the axes, and changing tools. Automatic state is the state in which the controller executes control plans (NC programs). During startup and shutdown modes, the automatic execution state would allow operator-free startup and shutdown of the controller, while in manual mode the operator would be assisting these operations, perhaps by manually releasing tools or operating fixtures. Failure recovery and maintenance modes are modes in which the automatic state does not apply.

\subsubsection{Resource States}

Resources include such items as coolant and other consumables, tooling, part loaders, and fixtures. The WMSA maintains the state of any interlocks or semaphores which limit access to resources by the operator interface, other DIAs, and control plans. In workstations with several CSAs, such as those consisting of a robot and machine tool, arbitrating requests for shared resources is an important function of the WMSA.

\subsection{WMSA Events}

In order for Domain Independent Applications to easily act on the modes and states of the controller, there needs to be the provision for sending events to these DIAs. Controller events are akin to the events which drive graphical user interfaces, such as $\mathrm{X}$ Windows, and relieve the programmer of the burden of continually reading the controller to determine these states. These events include:

- notification of the transition from one mode to another, for the controller

- notification of the transition from one state to another, for each SA and module

- notification of Virtual Machine events, such as interrupts

- release of system semaphores, and system clock ticks

Provisions must be made for programmers to attach to each of these events discriminately, so that each program is not overloaded with inappropriate events.

\section{The Controls Standardized Application (CSA)}

The WMSA communicates via NML message passing to Controls Standardized Applications. The CSAs include the functions which are normally associated with standalone CNCs, namely part program interpretation, trajectory generation, execution of Programmable Logic Controller (PLC) programs, spindle control, and servo control of machine axes. For each machine, there is a single CSA; that is, in a workstation consisting of a three-axis vertical machining center and a parthandling robot, there would be a single WPSA, one WMSA, and two CSAs, one each for the robot and machine tool. Each CSA communicates via NML message passing to the single WMSA for the workstation. 
Because of the wide availability of products which perform some of the functions of the CSA mentioned above (such as motion control boards), the CSA defined in the SOSAS will be refined further into these modules: Plan Interpretation, Trajectory Generation, Discrete Input/Output, and Servo Control.

\subsection{The Plan Interpretation Module}

The Plan Interpretation Module, or interpreter, reads and executes Control Plans expressed in the SOSAS Neutral Command Language (NCL), or legacy languages such as EIA-274-D, BCL, or APT. The interpretation is the result of an NML command issued by the WMSA. This module is extensible, so that new statements may be added to the planning language to give part programmers the ability to reference enhancements. The interpreter reads control plans and issues the appropriate commands to its subordinates, the Discrete Input/Output Module and the Trajectory Generator Module.

The plan interpreter is the transition point between the abstract geometric part description and the physical realities of the machining workstation. The interpreter is responsible for generation of tool paths to achieve removal of some volume of material. In the RCS architecture, the plan interpretation module comprises the functions of both the Task and Elementary Move (EMOVE) levels. More specifically, in cases where the planning language contains macros (e.g., the EIA-274-D canned cycles), the primitives which make up the macro map to Elementary Move plans, while the macro itself maps to Task level plans.

In general, a CSA plan interpreter is concerned with coordinating machine motion with tool cutting. This coordinated effort results in tool-path motion keyframe poses to the trajectory generator and accessory commands to devices such as a tool changer or gripper. All motion pathways must also be checked for the clearance of tools and machine links with obstacles.

\subsubsection{Plan Interpretation Functionality}

The required capabilities of the plan interpreter module include:

- interface to the Discrete Input/Output Module (e.g., tool change requests)

- interface to the Trajectory Generation Module (e.g., linear and circular interpolation)

- interpretation of part programs (e.g., EIA-274-D, BCL, or NCL languages)

- cuing the machinist for part fixturing and other manual tasks

- path-planning based on part features

- e nsion of the interpreter to handle instructions which may result from enhancements of the subordinate modules

- availability to DIAs of the identifiers of the currently executing Control Plan, the statement being executed now, and the statement which will be executed next

- availability to DIAs of the coordinate frame referenced by the current instruction

- availability to DIAs of the fixturing information

- availability to DIAs of the tooling information

- ability of DIAs to insert control plan statements

\subsubsection{Plan Interpretation Interfaces}

The plan interpreter communicates with the Discrete Input/Output Module and the Trajectory Generation Module of its CSA by passing messages which correspond to NCL statements in the control plan. If the control plans are not written in NCL (for example, EIA-274-D or BCL), the control plan statements need to be mapped into NCL messages. 
The NCL messages between the plan interpreter and the Discrete Input./Output Module are tabulated below. In this and other tables, the arguments in each message have been omitted for brevity, but will be detailed in documents which will follow this overview.

Table 1: Plan Interpretation to Discrete Input/Output NCL Messages

\begin{tabular}{|l|l|}
\hline NCL Message & Description \\
\hline \hline set_output & set an I/O point output \\
\hline get_input & get an I/O point input \\
\hline change_tool & change the tool \\
\hline activate_coolant & turn coolant on or off \\
\hline activate_spindle & turn the spindle on or off \\
\hline
\end{tabular}

The NCL messages between the plan interpreter and the Trajectory Generator Module are tabularized below:

Table 2: Plan Interpretation to Trajectory Generation NCL Messages

\begin{tabular}{|l|l|}
\hline NCL Message & Description \\
\hline \hline position_axes & non-coordinated axis move \\
\hline position_tool & non-coordinated tool move \\
\hline Iinear_move & coordinated axis linear move (EIA-274-D “G0” instruction) \\
\hline circular_move_cW & coordinated axis clockwise circular move (“G1") \\
\hline circular_move_ccW & coordinated axis clockwise circular move (“G2”) \\
\hline move_NURBS_curve & coordinated axis NURBS curvilinear move \\
\hline jog_axes & manual axis jog \\
\hline jog_tool & manual tool jog \\
\hline
\end{tabular}

\subsection{The Discrete Input/Output Module}

The Discrete Input/Output Module is responsible for executing plans targeted at discrete actuators such as valves and solenoids, and reading the state of sensors such as limit switches. These plans are typically called Programmable Logic Control programs, written in ladder logic or other highlevel languages such as those specified in the IEC-1131 standard (e.g., Grafcet), BASIC, or C. Requests for the execution of these plans are issued by the interpreter, during the course of the interpretation of a control plan. In the RCS architecture, the discrete input/output module performs Primitive (PRIM) level functions, specifically those concerned with discrete actuator control.

Discrete input/output plans are loosely coupled with motion control and will be separated from motion control to insure modularity. In some cases, however, a discrete I/O device may be loosely coupled to axis motion while the machine is operating in one mode, and tightly coupled in another mode. For example, spindle rotation is normally controlled independently from axis motion, and is merely maintained at a constant velocity for machining operations and changed infrequently. However, during tapping, the rotation of the spindle must be tightly coordinated with the $\mathrm{Z}$ axis, 
and control of the spindle needs to be dynamically reconfigurable so that it may be coordinated with the axis trajectories.

\subsubsection{Discrete Input/Output Functionality}

The required capabilities of the Discrete Input/Output module include:

- ability to run PLC programs written in one of the IEC-1131 languages, or the C language, as specified by the SOSAS

- availability to DIAs of the current values of each of the sensors and actuators to which the Discrete Input/Output module is interfaced

\subsubsection{Discrete Input/Output Interfaces}

The Discrete I/O CSA module is connected to the external actuators and sensors through standard interfaces. De facto interfaces, such as 0 to 24 volt or 4 to 20 milliamp references, are typical and will be supported. Standards for interfacing a variety of devices, such as the OBIOS from the Real Time Consortium, or CAN Bus, will also be supported. Additionally, the Discrete Input/Output module may have subordinate Servo Control modules, for example when controlling spindle velocity. The nature of this interface is somewhat ill-defined at the moment, so no attempt will be made to differentiate the functionality of the subordinate servo module or its interfaces from those of the Discrete Input/Output module at this time.

Table 3: External Discrete Input/Output Module Interfaces

\begin{tabular}{|l|l|}
\hline Interface & Description \\
\hline \hline 0 to 24 Volt & typical digital I/O point \\
\hline 0 to 5 Volt & typical analog or digital I/O point \\
\hline CAN Bus & Philips discrete input/output bus \\
\hline OBIOS & Real Time Consortium open basic input/output system \\
\hline
\end{tabular}

\subsection{The Trajectory Generator Module}

The Trajectory Generation Module is responsible for transforming motion path points into dynamic curvilinear trajectories. The motion path points are passed to the trajectory generator by the interpreter during control plan interpretation. The generation of dynamic curvilinear trajectories typically involves computing smooth motion in Cartesian or other world coordinate space. Points along the computed smooth trajectory are selected periodically at the interpolation interval, and sent to the Servo Control Module. In the RCS architecture, these functions are performed by the Primitive (PRIM) level, in cases where the actuators are continuously controlled and tightly coordinated.

In a normal machining operation the execution is open-loop, during which a series of position and velocity setpoints are "picked off" the trajectory in world coordinates and then sent to the Servo Control module. However, in many cases the generation of trajectories requires the use of sensor feedback, as is the case with guarded moves or constant-force machining.

The actual conversion of trajectories from the world coordinate system to joint space takes place in the Servo Control module. This is a requirement when performing Cartesian servoing, for 
example, in which servo computations are done in world coordinates. The responsibility for making available the kinematics and Jacobian are thus placed upon the Servo Control module.

\subsubsection{Trajectory Generation Module Functionality}

The required capabilities of the Trajectory Generation module include:

- ability to plan and execute linear and circular trajectories

- the ability to extend the trajectory planning to handle trajectories defined with NURBS

- the ability to offset trajectories with transforms generated by sensor data

- availability to DIAs of the current world coordinate frame used for trajectory calculations

- availability to DIAs of the current position and velocity of the tool tip in the world coordinate frame

- availability to DIAs of the timer semaphore which defines the interpolation interval

\subsubsection{Trajectory Generation Interfaces}

The Trajectory Generation Module communicates with the Servo Control Module of its CSA by passing messages which contain the computed setpoints for the tool position, in the current world coordinate frame. Additionally, configuration control message are passed to the servo controller. These messages corresponds to NCL statements, and are tabularized below:

Table 4: Trajectory Generation to Servo Control NCL Messages

\begin{tabular}{|l|l|}
\hline NCL Message & Description \\
\hline \hline set_axis_limits & define axis limits \\
\hline axis_position & position setpoint \\
\hline axis_velocity & velocity setpoint \\
\hline
\end{tabular}

\subsection{The Servo Control Module}

The Servo Control Module of the CSA is responsible for device interface for axis control and sensing of device feedback. The servo controller computes the motions of the individual joint actuators required to move the tool to the setpoints issued by the trajectory generator. Typically, the servo control module calculates actuator set points at a higher frequency than they are sent by the trajectory generator, so that subinterpolation needs to be performed. While controlling the device, the servo level is responsible for monitoring and interpreting the device feedback, computing outputs to the servoed axes using PID or other control laws. The servo control module corresponds to the Servo level of the RCS architecture.

\subsubsection{Servo Controller Functionality}

The required capabilities of the Servo Control module include:

- transforming world coordinate system (e.g., Cartesian points, ZYZ Euler angles) into the joint coordinate system

- subinterpolation of actuator setpoints

- initialization and monitoring of the axis controllers

- availability to DIAs of the current values of each of the sensors and actuators to which the servo module is interfaced

- ability of DIAs to access the external servo controller interfaces 


\subsubsection{Servo Controller External Interfaces}

The Servo Control CSA module is connected to the external actuators and sensors through standard interfaces. De facto interfaces, such as quadrature encoder and $+/-10$ volt axis velocity references, are typical and will be supported. Standards for interfacing a variety of devices, such as the OBIOS from the Real Time Consortium, or SERCOS, will also be supported.

Table 5: External Servo Control Module Interfaces

\begin{tabular}{|l|l|}
\hline Interface & Description \\
\hline \hline quadrature encoding & incremental feedback from encoders \\
\hline$+/-10$ volt & velocity reference to motor amplifiers \\
\hline PWM & velocity reference to motor amplifiers \\
\hline OBIOS & Real Time Consortium open basic input/output system \\
\hline
\end{tabular}

\section{The Virtual Machine}

The Virtual Machine is the set of all software which interfaces directly to the underlying controller hardware. While the operating system provides most of this interface, such as the file system, more is required to support the additional capabilities of a machine tool or robot. The additional interfaces which comprise the Virtual Machine are the communication system, the display interface, and the information base.

\subsection{The Operating System}

The operating system is specified by the SOSAS to be POSIX-compliant. POSIX does not specify a particular operating system, but the interfaces to systems programmers that operating systems must provide. In our Shop Floor Controller, the current operating system is DOS, which is not POSIX compliant; in the Laboratory Development Controller we are running LynxOS, which is a real-time POSIX-compliant operating system.

It is anticipated that full support of POSIX functionality (let alone exact compliance) will not be required for a machine controller, but that a subset is more appropriate. Part of the purpose of the implementation phase of the EMC project, at least as far as the Laboratory Development Controller is concerned, is to define that subset. At this point, it is clear that at a minimum, we need the functionality of the POSIX real-time extensions for semaphores, shared memory, clocks and timers, interprocess communication (see the next section), and threads. However, we feel that it is inappropriate and unnecessary to declare that the operating system be POSIX-compliant, in the same way that we would not declare that controllers use the VME bus. Other suitable and popular operating systems exist that we feel vendors will select based on their market. While this complicates things for developers (e.g., porting and support for both the Macintosh and PC), it is certainly an improvement over the proliferation of unique operating systems or lack thereof that characterizes today's controller market.

\subsection{The Communication System}

While POSIX specifies several mechanisms for interprocess communication (for instance, message queues) what is needed is a uniform interface to communication that can be configured for a particular mechanism. NIST has implemented a unique communication system which 
presents a programming interface for sending, testing, and receiving messages between two separate processes. This communication system interface is identical to programs regardless of which operating system or which hardware on which the programs run. The actual mechanism underlying the reads, tests, and writes is dynamically configurable to several types which are analogous to the Unix block special or character special devices. Block special mechanisms do not support queueing of data; that is, new data overwrites old data regardless of whether it has been read. These mechanisms are suitable for messages which upon arrival supercede any messages which arrived before. Character special mechanisms queue the messages. These mechanisms are suitable for messages which assume that previous messages have been received and that their actions will occur. NIST is in the process of defining these mechanisms so that they are implemented using POSIX calls.

Pursuant to the NIST RCS [2, 3], we recommend that all communication mechanisms use the overwriting protocols, since queueing can be implemented on top of these using agreed-upon process-to-process handshaking with no loss of generality. The advantage of the overwriting protocols is that they naturally fit into the framework of cyclic closed-loop control, the model used for all levels in the RCS hierarchy.

\subsection{Display System}

The display system provides developers with a way to display data to the operator and to get input from the operator. The display system will probably be most heavily used by the Operator Interface DIA. Defining the display system allows developers of machine tool and robot controller user interfaces to write portable code that can be used on a variety of different machines.

The SOSAS specifies that X Windows and Motif provide the display services. Recently, this requirement has been relaxed, so that other display systems such as Microsoft Windows or Open Look can be considered. This complicates things for the user interface developer, but gives additional flexibility.

\subsection{Information Base}

The information base provides a uniform interface for requests to store or fetch information about the state of the controller. This information is distributed across the controller (for example, axis positions in the servo module, kinematics in the trajectory module, tool ID in the discrete input/output module). It is too cumbersome to require that each module copy public data to a single information base. Rather, request are made to the individual module for information. These requests can be implemented so that they minimize the data latency. For example, if fast access to servo control data is required, the information base functions would be compiled in to the servo module, and all public data would appear in its address space. Alternatively, if the requesting program does not need fast access to the data, it may reside outside the module. In this case, the information base functions would be implemented using network transport mechanisms.

\section{Safety}

When defining the architecture of an open control system, one which will allow non-cooperating vendors to provide products and enhancements to an existing controller, some questions arise:

- What guarantees that the added product cannot damage the machine or injure the operator?

- Who is liable in case this happens?

In answering the first question, it should be noted that safety can never be absolutely guaranteed. Whatever mechanisms the controller or machine builders provide, the machinist can always find a 
way to circumvent them. Limit switches which detect the removal of safety shields can be taped shut by machinists. Dead man's switches can be clamped closed. Any protection in software is vulnerable to the same attacks used to override copy protection. Code developers can remove nuisance safety mechanisms by simply overwriting the code with "no-ops," or null operations. Safety programs can simply fail to work due to insufficient testing. Processors executing the safety software may fault, simultaneously causing a dangerous axis runaway condition while halting the software which could check for this.

A solution to these problems is to incorporate a "watchdog" safety system [8] into the controller, consisting of completely separate hardware which constantly monitors the activity of the workstation and causes a complete but safe shutdown when any safety conditions are violated. Examples of conditions to be monitored include

- axes position, velocity, and acceleration

- tool speed

- cutter force

- engagement of safety shields

- servo following error

- motor current and torque

- hardware health signals ("heartbeats") of computers and equipment

- software health signals of processes

When safety conditions are violated, the watchdog safety system opens the emergency stop loop circuitry for a full stop of all systems which could potentially cause damage to the machine or operator, such as axes, spindle, and tooling. The connection of these to the emergency stop loop must be made so that they are not damaged when such a stop is made.

The watchdog safety system must exist as a separate system which is not intended to be extensible. The conditions to be monitored should be set at limits which no reasonable machining operation would ever exceed. Limits are only set by the OEM. The conditions must be available to the watchdog safety system in "raw" form, i.e., not after processing by the controller software.

For example, axes position and velocity may be captured directly from encoders, scales, or tachometers, and fed to the safety system. Derived quantities such as acceleration must be computed by the safety system, not the machine controller, by hardware or by software which is simple and correct in the software engineering sense.

Health signals, or "heartbeats," should be provided by vital hardware (such as the CPU) in the form of continuously alternating signal levels, not absolute levels. The absence of alternation is an indication that the hardware has failed. Software processes (such as the looping of Discrete Input/Output module processes) should generate hardware signals which also alternate in level to indicate health. The watchdog safety system can be equipped with missing pulse detectors to trigger a fault when these processes have failed to issue an alternating signal.

\section{Road Map of Subsequent Documents}

This document is an initial overview of the architecture that the EMC engineers will use as the reference for controller development. Machine controllers will be built which conform to this reference model architecture. As a result of the implementation efforts, details of the standardized applications and modules will become clear, and will form the foundation of a second series of documents. Listed below are each of the documents which will be issued, and a brief description of their intended contents. 


\section{Workstation Planning Standardized Application}

This document covers the WPSA, focusing on two areas: scheduling and programming. Scheduling will discuss how the workstation connects to the rest of the factory network, what communication protocols are used (e.g., MMS), and what is responsible for scheduling jobs in the factory.

\section{Workstation Management Standardized Application}

This covers the WMSA "engine," and details the minimum capabilities required for a controller to be a controller. In this document we will discuss the modes and states of the controller.

\section{Plan Interpretation Module}

Here will be detailed the planning languages (e.g., EIA-274-D, BCL, NCL), and how we implemented extensibility, i.e., the ability to add a new $\mathrm{G}$ code to reflect the addition of NURBS profiling in the trajectory generator. We will also detail the interfaces that allowed us to "swap" the plan interpretation module.

\section{Discrete Input/Output Module}

PLC languages will be detailed here, as will the messages that run PLC programs. IEC-1131 will be addressed in this document.

\section{Trajectory Generation Module}

The bulk of the trajectory generation module will derive from the more complex realm of five-axis milling and robotics.This is where we will detail the commands used to build a queue of motion primitives, and what those primitives are. Extensibility will be demonstrated by adding NURBS trajectory generation to the weak set which is normally used now, namely straight lines and circles, and we'll tell how we did it. What we used to represent the machine's kinematic model, Jacobian, and other models will be spelled out.

\section{Servo Control Module}

This document will contain the list of all servo parameters we'll need, based on position control but with the flexibility to handle force and other physical quantities as well. Details of the error compensation methods we used (thermal/geometric, lead screw compensation tables, etc.) will appear in this volume. The hardware and software interfaces we defined which allow us to replace motion control boards from one vendor with those of another will be written down.

\section{Virtual Machine}

The controller "infrastructure" will be laid out here. This includes the operating system, display capability, control panel interface, system variables, and events. How we handled different buses and CPUs will be detailed.

\section{Domain Independent Applications}

The domain-independent applications include the operator interface, tool management, part probing, and thermal compensation. The interfaces which each required will be documented, as will the mechanism by which each was performed. 


\section{References}

1. The Martin Marietta Corporation, Next Generation Workstation/Machine Controller Specification for an Open System Architecture Standard (Draft), Volumes I through VI, Document No. NGC-0001-13-000-SYS, March 1992.

2. Albus, J. S., "RCS: A Reference Model Architecture for Intelligent Control," IEEE Journal on Computer Architectures for Intelligent Machines, May 1992.

3. Albus, J. S., Lumia, R., Fiala, J. C., and Wavering, A. J., "NASREM: The NASA/NBS Standard Reference Model for Telerobot Control System Architecture," Proceedings of the 20th International Symposium on Industrial Robots, Tokyo, Japan, October 4-6, 1989.

4. POSIX (Portable Operating System Interface), FIPS Publication 151-1.

5. Simpson, J., Hocken, R., and Albus, J., "The Automated Manufacturing Research Facility of the National Bureau of Standards," Journal of Manufacturing Systems 1 (1), 1983.

6. Kramer, T., "Process Planning for a Milling Machine from a Feature-Based Design," Proceedings of Manufacturing International, Atlanta, Georgia, April 1988, ASME, Vol. III, pp. 179-189, 1988.

7. National Computer Graphics Association, IGES/PDES Organization Reference Manual, January 1993. NCGA: 2722 Merrilee Drive, Suite 200, Fairfax, VA 22031.

8. R. D. Kilmer, H. G. McCain, M. Juberts and S. A. Legowik, "Safety Computer Design and Implementation," International Trends in Manufacturing Technology: Robot Safety, IFS (Publications) Ltd., Springer-Verlag, Berlin, 1985. 

